\title{
Non-Commutative Quantum Mechanics
}

\author{
J. Gamboa ${ }^{1 *}$, M. Loewe ${ }^{2 \dagger}$ and J. C. Rojas ${ }^{3 \ddagger}$ \\ ${ }^{1}$ Departamento de Física, Universidad de Santiago de Chile, Casilla 30\%, Santiago 2, Chile \\ ${ }^{2}$ Facultad de Física, Pontificia Universidad Católica de Chile, Casilla 306, Santiago 22, Chile \\ ${ }^{3}$ Departament ECM, Facultat de Fisica, Universitat de Barcelona and Institut D'Altes Energies, \\ Diagonal 647, E-08028, Barcelona, Spain
}

A general non-commutative quantum mechanical system in a central potential $V=V(r)$ in two dimensions is considered. The spectrum is bounded from below and for large values of the anticommutative parameter $\theta$, we find an explicit expression for the eigenvalues. In fact, any quantum mechanical system with these characteristics is equivalent to a commutative one in such a way that the interaction $V(r)$ is replaced by $V=V\left(\hat{H}_{H O}, \hat{L}_{z}\right)$, where $\hat{H}_{H O}$ is the hamiltonian of the two-dimensional harmonic oscillator and $\hat{L}_{z}$ is z- component of the angular momentum. For other finite values of $\theta$ the model can be solved by using perturbation theory.

Recent results in string theory [1] suggest that the spacetime could be non-conmutative [2]. This intriguing possibility implies new and deep changes in our conception of spacetime that could be visualized at the quantum mechanical level. For example, unitarity in quantum mechanics is assured if time is commutative, but the spatial non-commutativity, although it is completely consistent with the standard rules of quantum mechanics, imply the new Heisenberg relation

$$
\Delta x \Delta y \sim \theta,
$$

where $\theta$ is the strength of the non-commutative effects and plays an analogous role to $\hbar$ in usual quantum mechanics.

In this letter we would like to discuss a general non-commutative quantum mechanical system stressing the differences with the equivalent commutative case. More precisely, we show that any two-dimensional noncommutative system in a central potential $V=V(r)$ where $r=\sqrt{|\mathbf{x}|^{2}}$ is equivalent to a commutative system decribed by the potential

$$
V=V\left(\hat{H}_{H O}, \hat{L}_{z}\right)
$$

where $\hat{H}_{H O}$ is the hamiltonian of the usual (commutative) two-dimensional harmonic oscillator and $\hat{L}_{z}$ is the z-component of the angular momentum.

*E-mail: jgamboa@lauca.usach.cl

${ }^{\dagger}$ E-mail: mloewe@fis.puc.cl,

${ }^{\ddagger}$ E-mail: rojas@sonia.ecm.ub.es
In the non-commutative space one replace the ordinary product by the Moyal or star product

$$
\mathbf{A} \star \mathbf{B}(\mathbf{x})=\left.e^{\frac{i}{2} \theta^{i j} \partial_{i}^{(1)} \partial_{j}^{(2)}} \mathbf{A}\left(\mathbf{x}_{1}\right) \mathbf{B}\left(\mathbf{x}_{2}\right)\right|_{\mathbf{x}_{1}=\mathbf{x}_{2}=\mathbf{x}} .
$$

The only modification to the Schrödinger equation

$$
i \frac{\partial \Psi(\mathbf{x}, t)}{\partial t}=\left[\frac{\mathbf{p}^{2}}{2 m}+V(\mathbf{x})\right] \Psi(\mathbf{x}, t),
$$

is to replace

$$
V(\mathbf{x}) \star \Psi(\mathbf{x}, t) \rightarrow V\left(\mathbf{x}-\frac{\tilde{\mathbf{p}}}{2}\right) \Psi,
$$

where $\tilde{p}_{i_{k}}=\theta^{i_{k} j_{k}} p_{j_{k}}$, being $\theta_{i j}=\theta \epsilon_{i j}$ with $\epsilon_{i j}$ the antisimetric tensor. This formula that appeared lately in connection with string theory was written in [3] although there is an older version also known as Bopp's shift [4].

The next step is to consider a central potential in two dimensions. The right hand side of (5) becomes

$$
\begin{aligned}
V\left(\left|\mathbf{x}-\frac{\tilde{\mathbf{p}}}{2}\right|^{2}\right) \Psi & =V\left(\frac{\theta^{2}}{4} p_{x}^{2}+x^{2}+\frac{\theta^{2}}{4} p_{y}^{2}+y^{2}-\theta L_{z}\right) \Psi \\
& =V(\hat{\aleph}) \Psi
\end{aligned}
$$

where the $\hat{\aleph}$ operator is defined as

$$
\hat{\aleph}=\hat{H}_{H O}-\theta \hat{L}_{z},
$$

and corresponds to a two-dimensional harmonic oscillator with effective mass $m=2 / \theta^{2}$, frequency $\omega=\theta$ and angular momentum $L_{z}=x p_{y}-y p_{x}$. The symmetry group for this system is $S U(2)$ and the spectrum of $\hat{\aleph}$ can be computed noticing that

$$
\begin{aligned}
L_{x} & =\frac{1}{2}\left(a_{x}^{\dagger} a_{x}-a_{y}^{\dagger} a_{y}\right), \\
L_{y} & =\frac{1}{2}\left(a_{x}^{\dagger} a_{y}+a_{y}^{\dagger} a_{x}\right), \\
L_{z} & =\frac{1}{2 i}\left(a_{x}^{\dagger} a_{y}-a_{y}^{\dagger} a_{x}\right),
\end{aligned}
$$

are symmetry generators satisfying the Lie algebra $\left[L_{i}, L_{j}\right]=i \epsilon_{i j k} L_{k}$ and, therefore, $\left\{\hat{\aleph}, \hat{\mathbf{L}}^{2}, J_{z}=\frac{1}{2} \hat{L}_{z}\right\}$ is a complete set of conmuting observables. If we denote by $\lambda_{j m}$ and $\mid j, m>$ the eigenvalues and eigenvectors, respectively, then we have the selection rules

$$
\begin{aligned}
j & =0, \frac{1}{2}, 1, \frac{3}{2}, \ldots \\
m & =j, j-1, j-2, \ldots,-j .
\end{aligned}
$$


The eigenfunctions $\mid j, m>$ are well known [5] and the eigenvalues of $\hat{\aleph}$ are given by

$$
\lambda_{j m}=\theta[2 j+1-2 m] .
$$

Using these results, the calculation of the eigenvalues of $V(\hat{\aleph})$ is straightforward. Indeed, if the eigenvalues of the operator $\hat{A}$ are $a_{n}$, then the function $f(\hat{A})$, after expanding for small values of $\epsilon$, is

$$
\begin{aligned}
f(\hat{A}+\epsilon) \psi_{n} & =\left(f(\hat{A})+f^{\prime}(\hat{A}) \epsilon+\frac{1}{2 !} f^{\prime \prime}(\hat{A}) \epsilon^{2} \cdots\right) \psi_{n} \\
& =\left(f\left(a_{n}\right)+f^{\prime}\left(a_{n}\right) \epsilon+\frac{1}{2 !} f^{\prime \prime}\left(a_{n}\right) \epsilon^{2} \cdots\right) \psi_{n} \\
& =f\left(a_{n}+\epsilon\right) \psi_{n} \rightarrow f\left(a_{n}\right) \psi_{n},
\end{aligned}
$$

and, as a consequence, the eigenvalue equation of $V(\hat{\aleph})$ is

$$
V(\hat{\aleph})|j, m>=V[\theta(2 j+1-2 m)]| j, m>.
$$

Once equation (12) is found, one must compute the spectrum of the full hamiltonian given by

$$
\begin{aligned}
H & =\frac{\mathbf{p}^{2}}{2 M}+V(\hat{\aleph}) \\
& =\frac{2}{M \theta^{2}}\left(\frac{\theta^{2}}{4} \mathbf{p}^{2}+\mathbf{r}^{2}-\theta L_{z}\right)-\frac{2}{M \theta^{2}} \mathbf{r}^{2}+V(\hat{\aleph})+\frac{2}{M \theta} L_{z} \\
& =\frac{2}{M \theta^{2}} \hat{\aleph}+V(\hat{\aleph})-\frac{2}{M \theta^{2}} \mathbf{r}^{2}+\frac{2}{M \theta} L_{z} \\
& \equiv H_{0}-\frac{2}{M \theta^{2}} \mathbf{r}^{2}+\frac{2}{M \theta} L_{z} .
\end{aligned}
$$

By using equations (10) and (12) one find that the eigenvalues of $\hat{H}_{0}$ are

$$
\Lambda_{j, m}=\frac{2}{M \theta}[2 j+1-2 m]+V[\theta(2 j+1-2 m)] .
$$

The second term of the Hamiltonian can be treated as a perturbation for large (but finite) values of $\theta$. For regular, polynomial-like potentials, the situation is similar to the solitonic case as in [6].

We will concentrate on the expectations values of the full Hamiltonian, $E_{j m}=\langle j, m|\hat{H}| j, m\rangle$ i.e.

$$
\begin{aligned}
E_{j m} & =<j, m\left|\hat{H}_{0}\right| j, m>-\frac{2}{M \theta^{2}}<j, m\left|\mathbf{r}^{2}\right| j, m> \\
& +\frac{4}{M \theta}<j, m\left|L_{z}\right| j, m>, \\
& =\frac{2}{M \theta}[2 j+1]+V[\theta(2 j+1-2 m)] \\
& -\frac{2}{M \theta^{2}}<j, m\left|\mathbf{r}^{2}\right| j, m>.
\end{aligned}
$$

The next to last term in the right hand side in (15) can be calculated using perturbation theory for large values of $\theta$. Indeed, in such case $\mid j, m>$ corresponds to the two-dimensional harmonic oscillator eigenvectors

$$
\left|j, m>=\frac{a_{+}^{\dagger+m} a_{-}^{\dagger j-m}}{\sqrt{(j+m) !(j-m) !}}\right| 0,0>,
$$

where in (16) we have used the Schwinger representation for the two- dimensional harmonic oscillator [5] and $<j, m\left|\mathbf{r}^{2}\right| j, m>$ becomes

$$
<j, m\left|\mathbf{r}^{2}\right| j, m>=\frac{\theta}{2}[2 j+1] .
$$

Let us consider now two kinds of singular potentials.

a)

If $V(r)=-\gamma / r^{\alpha}$, then $V(\theta)=-\gamma[\theta(2 j+1-2 m)]^{-\frac{\alpha}{2}}$. Note that this term goes like $\theta^{-\frac{\alpha}{2}}$, and therefore, the relevant contribution to the spectrum, for $\alpha>2$ is given by the first term in $(15)$. In fact the difference between the levels $2 j$ and $2 j+1$ is $2 / M \theta$.

b) From the physical point of view, probably the most interesting case is the Coulomb potential $V(r)=\gamma \ln (r)$ which corresponds to $V(\theta)=\gamma / 2 \ln [\theta(2 j+1-2 m)]$. This could have a relation to the Quantum Hall Effect where electrons are confined in a plane. We would like to remark that spectroscopy in two dimensional systems could be a sensible mechanism for detecting noncommutative corrections to quantum mechanics [7].

Finally we would like to point out that our results seem to indicate that the connection between the commutative and non-commutative regimes is abrupt, i.e. the limit $\theta \rightarrow 0$ cannot be taken directly [8].

\section{ACKNOWLEDGMENTS}

We would like to thank V. O. Rivelles and F. Méndez for several discussions on non-commutative geometry. This work has been partially supported by the grants Nr. 1980788 and Nr. 1980577 from Fondecyt-Chile and Dicyt-USACH.

[1] A. Connes, M. Douglas and A. S. Schwarz, JHEP 9802:003 ( 1998); N. Seiberg and E. Witten, JHEP 09, 032 (1999); T. Yoneya, Prog.Theor.Phys. 103, 1081 (2000).

[2] The literature is very extense, some references are; T. Filk, Phys.Lett. B376, 53 (1996); R. Gopakumar, J. Maldacena, S. Minwalla and A. Strominger, JHEP 0006036 (2000); L. Alvarez-Gaumé and S. Wadia, hep- th/0006219; M. Hayakawa, Phys. Lett. 476, 431 (2000); C. P. Martin and F. Ruiz, hep-th/0007131; J. Gomis and T. Mehen, hep-th/0005129; I. Mociou, M. Popelov and R. Roibar, Phys. Lett. 489B, 390 (2000); J. Lukierski, P. Stichel and W. Zakrzewski, Ann Phys. 260, 224 (1997); K. Valavano, hep-th/0006245; V.P. Nair and A.P. Polychronakos, hepth/0011172. 
[3] D. Bigatti and L. Susskind, Phys.Rev. D62, 066004 (2000); L. Mezincescu, Star Product in Quantum Mechanics, hep-th/0007046; B. Morariu, A. P. Polychronakos, hep-th/0102157.

[4] see e.g, T. Curtright, D. Fairlie and C. Zachos Phys. Rev. D58, 025002 (1998); C. Zachos, J. Math. Phys. 41, (2000).

[5] G. Baym, Lectures on Quantum Mechanics, pag. 380, Benjamin (1974).

[6] R. Gopakumar, S. Minwalla and A. Strominger, JHEP 05,020 (2000).

[7] In this context see; J. Gamboa, M. Loewe and J. C. Rojas, hep-th /0101081; D. Bak , S. K. Kim, K. Su Soh, J. H. Yee, Phys. Rev. Lett. 85, 3087 (2000); ibid hep-th/0006087

[8] S. Minwalla, M. Van Raamsdonk and N. Seiberg, hepth/9912072. 